\title{
Duração da larvicultura sobre o desempenho posterior de juvenis de jundiá, Rhamdia quelen, recriados em tanques-rede
}

\author{
Hatchery duration influence on posterior performance of jundiá fingerlings, Rhamdia quelen, \\ reared in cages
}

\author{
Juan José Santinón ${ }^{\mathrm{I}}$ David Roque Hernández ${ }^{*}$ Sebastián Sánchez ${ }^{\mathrm{I}}$ Hugo Alberto Domitrovic ${ }^{\mathrm{I}}$
}

\section{RESUMO}

Realizou-se um experimento fatorial $3 \times 2$, com três repetições $(n=18)$, a fim de avaliar o crescimento e a sobrevivência de juvenis de jundiá (Rhamdia quelen) recriados em tanques-rede e dispostos em tanques externos até os 65 dias de vida. Os peixes utilizados provêm de um sistema de larvicultura intensiva, em que foram alimentados em laboratório com três rações diferentes até os 10 e 15 dias de vida, antes de serem transferidos para os tanques-rede. Uma vez nos tanques-rede, os peixes foram alimentados até a saciedade com ração balanceada comercial contendo $28 \%$ de proteína bruta. Ao final do ensaio, não foram observadas diferenças significativas entre os tratamentos para nenhum dos parâmetros estimados $(P>0,05)$. Da mesma maneira, os valores de crescimento e sobrevivência obtidos foram similares entre os grupos de peixes transferidos aos 10 e 15 dias de vida. Os resultados demonstram que a passagem das larvas de jundiá para os tanques-rede de recria aos 10 dias de vida seria o manejo mais recomendável, diminuindo os custos da alimentação e operativos que implicam o estágio de larvicultura sob condições controladas de laboratório.

Palavras-chave: juvenis, crescimento, larvicultura, densidade, tanques-rede, Rhamdia quelen.

\section{ABSTRACT}

A $3 \times 2$ factorial trial with three replicates $(n=18)$ was carried out aiming to evaluate Rhamdia quelen fingerling growth and survival rates, stocked in cages, in external ponds until attain 65 days of life. Fish used come from an intensive hatchery system, where were fed in laboratory with three different diets until attain 10 and 15 days old, before being transferred to cages. Once in cages, fishes were fed until satiation with commercial balanced diet containing $28 \%$ crude protein. At the end of the experience, there were no significant differences between treatments for any of the estimated parameters $(P>$
0.05). Similarly, the values of growth and survival obtained were similar between the groups of fish transferred with 10 and 15 days old. The results show that transference of 10 days old catfish larvae to the cages would be the more appropriate management, reducing feeding and operating costs that involves the larviculture under controlled laboratory conditions.

Key words: fingerlings, growth, hatchery, stocking density, cages, Rhamdia quelen.

\section{INTRODUÇÃO}

Durante a última década, a aquicultura demonstrou um crescimento constante, alcançando $50 \%$ da produção mundial da pesca marinha e continental (FAO, 2008). Vale ressaltar que o crescimento é determinado por estabelecimentos dedicados à produção de larvas e juvenis e empresas elaboradoras de rações e insumos aquícolas. Nesse sentido, um dos principais inconvenientes que retarda o avanço da aquicultura é a obtenção de larvas em grande escala, de boa qualidade e sua correta alimentação nos primeiros dias de vida (GARCIAORTEGA et al., 2001).

Nos sistemas de cria atuais, para a obtenção de juvenis, é comum a utilização de sistemas semiextensivos de larvicultura ao ar livre, intensivos em laboratório, ou mistos, combinando esseees dois (JOMORI et al., 2003). De maneira geral, uma vez que a abertura bucal das larvas é produzida, estas podem ser transferidas aos viveiros, tanques e tanques-rede ou

'Instituto de Ictiología del Nordeste, Facultad de Ciencias Veterinarias, Universidad Nacional del Nordeste, (UNNE), Sargento Cabral 2.139, Corrientes, Argentina. E-mail: dhernandez@vet.unne.edu.ar.*Autor para correspondência. 
mantidas em laboratório sob condições controladas com alimento vivo, ração balanceada ou uma combinação de ambos (BALDISSEROTTO \& RADÜNZNETO, 2004).

JOMORI et al. (2003) obtiveram altas porcentagem de sobrevivência em larvas de pacu (Piaractus mesopotamicus) ao longo de uma larvicultura de nove dias, em sistemas intensivos sob condições controladas, mas, ao serem transferidas aos tanques, mostraram apenas $54 \%$ de sobrevivência aos 45 dias de vida. Esses valores foram igualmente superiores quando comparados aos obtidos com aquelas larvas que foram transferidas aos tanques após a abertura bucal com apenas $11 \%$ de sobrevivência.

Segundo BALDISSEROTTO \& RADÜNZ NETO (2004), as larvas de jundiá mantidas sob condições controladas em 21 dias de vida podem atingir de 80 a $95 \%$ de sobrevivência e um bom crescimento nesse período. Os autores afirmam também que, quando as larvas são de maior tamanho, menor é a chance de predação no viveiro. Por outro lado, a permanência das larvas sob condições controladas aumenta consideravelmente os custos de produção (PERSONLE RUYET et al., 1993).

A piscicultura em tanques-rede é uma técnica muito utilizada pelo fato de que permite explorar cursos ou massas de água, os quais não poderiam ser utilizados nas formas práticas convencionais como viveiros. $\mathrm{O}$ uso de açudes, barragens e zonas costeiras de rios e mares compreendem os ambientes mais utilizados nesse tipo de cultivo (SCHMITTOU, 1993).

O sistema de produção em tanques-rede instalados em tanques, viveiros e canais geralmente é utilizado para a engorda dos peixes (LUCHINI, 1988). Esse sistema possibilita aumentar as densidades, aperfeiçoar o uso de alimentos artificiais e melhorar a eficiência de conversão alimentar, facilitando ainda o manejo de rotina, o monitoramento sanitário e a despesca final. Dentro das desvantagens dessa produção, encontram-se a deterioração da qualidade da água em razão das elevadas densidades de estocagem, as possíveis doenças que se dispersam rapidamente e são mais difíceis de tratar e a possível ruptura que pode levar à fuga dos peixes (BEVERIDGE, 1986; SWANN et al., 1994).

Diversos trabalhos foram orientados a avaliar o desempenho produtivo de larvas de jundiá dentro do primeiro mês de vida sob condições controladas (BEHR et al., 2000; ULIANA et al., 2001; CARDOSO et al., 2004; HERNÁNDEZ et al., 2006; HERNÁNDEZ et al., 2009a), mas poucos são os estudos sobre o momento adequado para transferir as larvas aos viveiros ou tanques de alevinagem, com objetivo de diminuir o tempo de larvicultura em laboratório, sem afetar o posterior desempenho produtivo dos peixes.

O objetivo do presente trabalho foi avaliar a influência da alimentação na larvicultura e da duração dessa fase do ciclo sobre o desempenho posterior dos peixes recriados em tanques-rede ao longo de 10 e 15 dias de larvicultura em laboratório.

\section{MATERIAL E MÉTODOS}

O estudo foi realizado nas instalações da aquicultura experimental do Instituto de Ictiología do Nordeste (INICNE) da Faculdade de Ciências Veterinarias (Corrientes, Argentina), a partir de um experimento com duas etapas, uma de larvicultura sob condições de laboratório e outra seguida com passagem de larvas a tanques-rede, onde foram recriadas até os 65 dias de vida.

Até o momento de serem transferidas, as larvas na larvicultura em aquários receberam três tratamentos alimentares diferentes (A, B e C) compostos por: $35 \%$ de ovo de peixe, ensilado biológico de carcaça de peixe ou fígado de frango cru, respectivamente. Cada ração continha também levedura de pão, lecitina de soja e premix vitamínicominerais.

Os tratamentos foram definidos segundo a alimentação recebida em laboratório e o tempo de passagem aos tanques-rede:

Primeira passagem

TA larvicultura intensiva por 10 dias com ração A e passagem a tanques-rede;

TB larvicultura intensiva por 10 dias com ração B e passagem a tanques-rede;

TC larvicultura intensiva por 10 dias com ração $\mathrm{C} \mathrm{e}$ passagem a tanques-rede.

Segunda passagem

TA larvicultura intensiva por 15 dias com ração A e passagem a tanques-rede;

TB larvicultura intensiva por 15 dias com ração B e passagem a tanques-rede;

TC larvicultura intensiva por 15 dias com ração $\mathrm{C} \mathrm{e}$ passagem a tanques-rede.

Para cada fase de transferência, foram utilizados nove tanques-rede de $1 \mathrm{~m}^{3}$ submergidos em três tanques de alvenaria de $16 \mathrm{~m}^{3}$ de capacidade e utilizadas 900 larvas (1.800 em total), em uma lotação de 100 larvas por unidade experimental. Na tabela 1, apresentam-se os pesos médios estimados para os exemplares com diferentes tratamentos alimentares no momento de cada transferência. 
Uma semana antes da passagem das larvas, os tanques foram fertilizados com alfafa na quantidade de $300 \mathrm{~g} \mathrm{~m}^{-2}$. Os peixes transferidos aos tanques-rede foram alimentados até a saciedade com ração comercial contendo $28 \%$ de proteína bruta. A ração foi oferecida às $9,12,15$ e 18 horas, durante os primeiros 20 dias, e duas vezes ao dia, até o final do ensaio, às 10 e 18 horas.

O efeito das variáveis em estudo foi avaliado a partir da Porcentagem de Sobrevivência $(\% \mathrm{~S}=$ (total de peixes vivos ao final do ensaio/total de peixes estocados)*100), do Peso Médio Final (PMF), da Biomassa Final $\left(\mathrm{B}=\mathrm{PM}^{*}\right.$ total de peixes vivos ao final do ensaio) e do Coeficiente de Crescimento Específico (CCE), parâmetro que foi estimado segundo a seguinte equação:

$$
\operatorname{CCE}\left(\mathrm{g} \mathrm{dia}^{-1}\right)=\frac{3\left(\mathrm{P}_{\mathrm{t}}^{0,33}-\mathrm{P}_{0}{ }^{0,33}\right)}{\mathrm{t}-\mathrm{t}_{0}}
$$

em que $\mathrm{P}_{0}$ e $\mathrm{P}_{\mathrm{t}}$ correspondem ao peso inicial e final do período de estudos, $\mathrm{t}-\mathrm{t}_{0}$ ao tempo em dias e 0,33 ao coeficiente de crescimento alométrico estimado para peixes (HEPHER, 1988). Esse coeficiente foi utilizado em razão do fato de que corrige as diferenças alométricas de crescimento associadas ao tamanho dos peixes, já que dentro dos grupos transferidos aos 15 dias existiam importantes diferenças de peso entre os exemplares provenientes dos diferentes tratamentos alimentares em aquários e aqueles estocados nos tanques-rede aos 10 dias de vida (Tabela 1).

Os parâmetros de qualidade de água foram mensurados com equipamento $\mathrm{YSI}\left(\mathrm{O}_{2}\right.$ e temperatura, diariamente) e HANNA ( $\mathrm{pH}$ e condutividade, semanalmente). Os resultados obtidos foram avaliados mediante Análises da Variância, a partir de um desenho completamente aleatório dentro dos grupos estocados aos 10 e 15 dias ( $\mathrm{n}=9$ em cada data). As comparações de médias de tratamentos foram realizadas mediante $o$ Teste de Tuckey.

Tabela 1 - Pesos médios das larvas de jundiá dos distintos tratamentos alimentares no momento de serem transferidas para os tanques-redes em cada etapa.

\begin{tabular}{lccc}
\hline \multicolumn{4}{c}{ Tratamentos } \\
\hline Etapa & A & B & C \\
Primeira & $26.7^{\mathrm{a}}$ & $11.26^{\mathrm{b}}$ & $16.93^{\mathrm{b}}$ \\
Segunda & $115,1^{\mathrm{a}}$ & $23,5^{\mathrm{c}}$ & $66,6^{\mathrm{b}}$ \\
\hline
\end{tabular}

Médias seguidas por letras diferentes indicam diferenças significativas entre os tratamentos $(\mathrm{P}<0,05)$.

\section{RESULTADOS E DISCUSSÃO}

Os parâmetros da qualidade da água avaliados se mantiveram dentro dos limites aceitáveis para a espécie (Tabela 2) (BALDISSEROTTO \& RADÜNZ NETO, 2004). As médias estimadas para os diferentes parâmetros zootécnicos analisados no experimento são apresentadas na figura 1 .

Dentro dos grupos transferidos aos 10 dias de vida, não foram observadas diferenças significativas para nenhum dos parâmetros estimados $(\mathrm{P}>0,05)$, evidenciando que a alimentação recebida pelas larvas em aquários durante a etapa prévia à alevinagem em tanque-rede não afetou seu desempenho posterior.

Por sua vez, a análise dos resultados obtidos para o lote transferido aos 15 dias de vida evideciou que os exemplares do TB obtiveram um CCE ligeiramente superior nos tanques-rede (Figura 1a). Isso é um fato importante, já que nesse tratamento os peixes apresentaram um peso inferior no momento de serem transferidos, culminando no ensaio com pesos e biomassas similares aos TA e TC (Figura 1b e c). Além disso, não foram encontradas diferenças entre os diferentes momentos de passagem (10 e 15 dias de vida) das larvas ao final do ensaio para nenhuma das variáveis analisadas.

O cultivo de larvas em sistemas intensivos aparece como uma prática de uso crescente em numerosas espécies de peixes. Embora essa metodologia de trabalho demande instalações adequadas para manter animais a densidades elevadas, assim como pessoal capacitado para realizar as tarefas de alimentação e limpeza, aumentando os custos de produção, estes são compensados pela melhora nas taxas de sobrevivência obtidas em comparação com a transferência dos exemplares aos tanques após da abertura bucal (JOMORI et al., 2005).

ATENCIO-GARCIA et al. (2003) avaliaram a influência da primeira alimentação na alevinagem do yamú, Brycon siebenthalae, estocadas imediatamente em viveiros após o início da alimentação exógena com aquelas estocadas depois de receber a alimentação com larvas de pirapitinga por 24 horas, sendo obtidos 13 e $74 \%$ de sobrevivência, respectivamente, aos 15 dias de vida. De modo similar, MAI \& ZANIBONI-FILHO (2005) mostraram que as larvas de dourado (Salminus brasiliensis) que permaneceram mais tempo em laboratório (quatro e seis dias) apresentaram taxas de sobrevivência e crescimento maiores que aquelas mantidas por zero e dois dias de larvicultura controlada. Neste estudo, não foram encontradas diferenças significativas nas taxas de sobrevivência em nenhum dos momentos de passagem das larvas aos tanquesrede. 
Tabela 2 - Valores médios e desvio padrão (DP) estimados para os diferentes parâmetros da qualidade da água obtidos durante a realização do ensaio.

\begin{tabular}{lc}
\hline Parâmetros Medidos & Promedio \pm DP \\
\hline Temperatura da Água $\left({ }^{\circ} \mathrm{C}\right)$ & $29,05 \pm 1,75$ \\
pH & $7,14 \pm 0,49$ \\
Oxigênio $\left(\mathrm{mg} \mathrm{L}^{-1}\right)$ & $7,88 \pm 2,01$ \\
Oxigênio $(\%$ saturação) & $98,7 \pm 19,7$ \\
Condutividade $\left(\mu \mathrm{S} \mathrm{cm}{ }^{-1}\right)$ & $146,7 \pm 26,5$ \\
\hline
\end{tabular}

BARCELLOS et al. (2004) testaram distintas densidades de estocagem de juvenis de jundiá em tanques-rede $\left(100,200\right.$ e 300 ind $\left.\mathrm{m}^{-3}\right)$ e observaram que os melhores parâmetros de crescimento, tais como ganância de peso e taxa de crescimento específico, foram obtidos com a menor densidade de estocagem, enquanto que a sobrevivência foi semelhante para todas as densidades estudadas. No presente ensaio, os tratamentos A e C evidenciaram ocorrência de crescimento e sobrevivência similar à encontrada por esses autores para iguais densidades de estocagem (Figura 1d).
Os ensaios de larvicultura realizados em jundiá por diferentes pesquisadores demonstraram que existem poucas diferenças entre as larvas quanto a parâmetros de crescimento dentro dos primeiros 10 dias de vida para qualquer tipo de alimento provado (PIAIA \& RADÜNZ NETO, 1997; BEHR et al., 2000; ULIANA et al., 2001; HERNÁNDEZ et al., 2009a). Nos resultados do presente estudo, não foram encontradas diferenças na transferência dos peixes aos 10 ou 15 dias de vida, sendo a primeira opção a mais recomendável, já que, ao diminuir a duração da fase de aquários, os custos operativos diminuem, assim como a possibilidade de aparição de patologias associadas ao incorreto desenvolvimento esquelético nos sistemas de larvicultura intensiva (CAHU et al., 2003; HERNÁNDEZ et al., 2010).

\section{CONCLUSÃO}

A transferência dos peixes com 10 dias de vida aos tanques-rede seria o manejo mais recomendável para o jundiá.


b
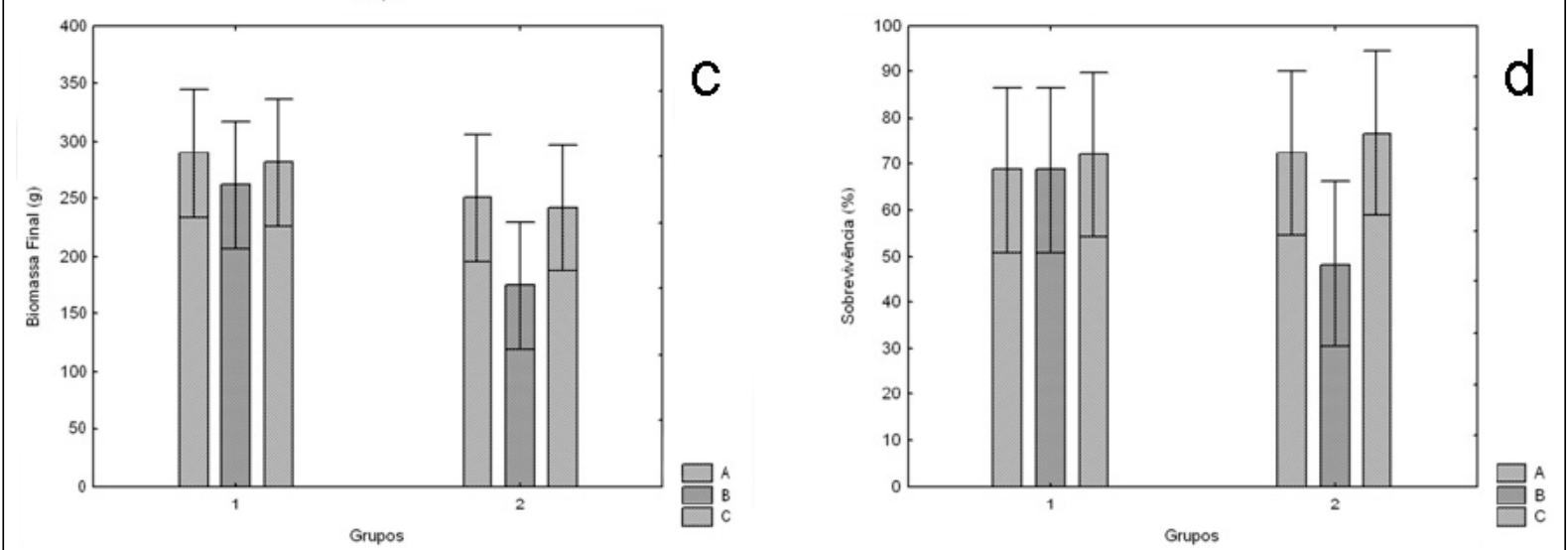

Figura 1 - Parâmetros de crescimento em juvenis de jundiá estimados após 65 dias de cultivo em tanques-rede. Os grupos 1 e 2 indicam os momentos de passagem das larvas. (a) Coeficiente de Crescimento Específico (\%); (b) Peso Médio Final (g); (c) Biomassa Final (g); e (d) Sobrevivência (\%). Os valores mostram intervalos de confiança (95\%). 


\section{AGRADECIMENTOS}

Os autores agradecem à bióloga Adriana Kloster as Universidade Federal do Acre (UFAC), pela ajuda fornecida na tradução do manuscrito, e ao financiamento obtido pelo PICT 13.332 e pela SGCyT (UNNE) PI 001/2007.

\section{REFERÊNCIAS}

ATENCIO-GARCÍA, V. et al. Influência da primeira alimentação na larvicultura e alevinagem do yamú Brycon siebenthalae (Characidae). Acta Scientiarum: Animal Science, v.25, n.1, p.61-72, 2003. Disponível em: http:// periodicos.uem.br/ojs/index.php/ActaSciAnimSci/article/view/ 2092/1429. Acesso em: 28 ago. 2009. doi: 10.4025/ actascianimsci.v25i1.2092.

BALDISSEROTTO, B.; RADÜNZ NETO, J. Criação de jundiá. Santa Maria: UFSM, 2004. 232p.

BARCELLOS, L.J.G. et al. Nursery rearing of jundiá, Rhamdia quelen (Quoy \& Gaimard) in cages: cage type, stocking density and stress response to confinement. Aquaculture, v.232, p.383394, 2004. Disponível em: <http://www.sciencedirect.com/science/ a r t i c 1 e/ B 6 T 4 D - 49 J H K Y 4 - 2/2/ f1214f5ec1d37ac5c313527a0acafb97>. Acesso em: 22 abr. 2005. doi:10.1016/S0044-8486(03)00545-3.

BEHR, E.R. et al. Ação do tempo e da forma de suplementação alimentar com Artemia franciscana sobre a sobrevivência e o crescimento de larvas de jundiá. Ciência Rural, v.30, n.3, p.503-507, 2000. Disponível em: <http://www.scielo.br/s c i e

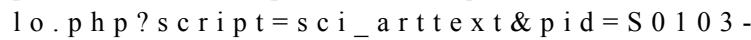
$84782000000300023 \& \operatorname{lng}=$ en $\& n r m=i s o \& t \operatorname{lng}=p t>$. Acesso em: 25 mar. 2006. doi: 10.1590/S0103-84782000000300023.

BEVERIDGE, M.C.M. Piscicultura en jaulas y corrales. Modelos para calcular la capacidad de carga y las repercusiones en el ambiente. República de Filipinas: FAO, 1986. 100p. (Documento Técnico de Pesca, 255).

CARDOSO, A.P. et al. Criação de larvas de jundiá (Rhamdia quelen) alimentadas com rações granuladas contendo fígados ou hidrolisados. Acta Scientiarum: Animal Sciences, v.26, n.4, p.457-462, 2004. Disponível em: <http://periodicos.uem.br/ ojs/index.php/ActaSciAnimSci/article/view/1718/1158>. Acesso em: 28 mar. 2006. doi: 10.4025/actascianimsci.v26i4.1718.

CAHU, C. et al. Nutritional components affecting skeletal development in fish larvae. Aquaculture, v.227, p.245-258, 2003. Disponível em: $<\mathrm{ht} \mathrm{t}$ p: / / w w w. s c i e n c e d r e c t. c o m / science?_ob=ArticleURL\&_udi=B6T4D-49CKXBH$1 \&$ _user $=1677679 \&$ \&coverDate $=11 \% 2$ F $10 \% 2$ F $2003 \&$ rdo $\mathrm{c}=1 \&$ \&mt $=$ high\&_orig $=$ search\&_sort $=\mathrm{d} \&$ _docanchor $=\& \mathrm{view}=\mathrm{c} \&$ _acct $=\mathrm{C} 000054197 \&$ \& version $=1 \&$ _urlVersion $=0 \&$ \&use rid $=1677679 \& \mathrm{md} 5=93 \mathrm{~b} 0510 \mathrm{~d} 596 \mathrm{c} 8 \mathrm{~b} 0 \mathrm{~b} 6 \mathrm{c} 4810 \mathrm{a} 6 \mathrm{c} 4 \mathrm{ad} 3288>$. Acesso em: 31 maio 2006. doi: 10.1016/S0044-8486(03)00507-6.

FAO. Examen mundial de la pesca y $\underline{\text { la }}$ acuicultura. Roma, 2008. 218p.
GARCÍA-ORTEGA, A. et al. Evaluation of protein quality in microbound started diets made with decapsulated cysts of Artemia and fish meal for fish larvae. Journal of the World Aquaculture Society, v.32, p.317-329, 2001. Disponível em: <http://dx.doi.org/10.1111/j.1749-7345.2001.tb00455.x>. Acesso em: 18 fev. 2007. doi: 10.1111/j.17497345.2001.tb00455.x

HERNÁNDEZ, D.R. et al. Evaluación de diferentes dietas en la primera alimentación del Bagre sudamericano (Rhamdia quelen). In: CONGRESO IBEROAMERICANO VIRTUAL DE ACUICUlturA, 4., 2006, Zaragoza, España. Anales... Zaragoza: CIVA, 2006. p.1151-1155.

HERNÁNDEZ, D.R. et al. Fontes não-convencionais de proteína na primeira alimentação do bagre sul americano (Rhamdia quelen). Ciência Rural, v.39, n.3, p.878-884, 2009a. Disponível em: <http://www.scielo.br/pdf/cr/v39n3/ a125cr653.pdf>. Acesso em: 28 jun. 2009. doi: 10.1590/ S0103-84782009005000019.

HERNÁNDEZ, D.R. et al. Incidence of bone malformations in the vertebral column and caudal skeleton of Rhamdia quelen larvae. Anatomia, Histologia, Embryologia, v.39, n.1, p.8791, 2010. (Abstract). Disponível em: <http://dx.doi.org/ 10.1111/j.1439-0264.2009.00984.x>. Acesso em: 20 jan. 2010. doi: 10.1111/j.1439-0264.2009.00984.x.

HEPHER, B. Nutrición de peces comerciales en Estanques. Dor, Israel: Fish and Aquaculture Research Station, 1988. $311 \mathrm{p}$.

JOMORI, R.K. et al. Growth and survival of pacu Piaractus mesopotamicus (Holmberg, 1887) juveniles reared in ponds or at different initial larviculture periods indoors. Aquaculture, v.221, p.277-287, 2003. Disponível em: <http:// www.sciencedirect.com/science/article/B6T4D-481FSP2-3/2/ 6f18a11db8df60b09f2194078107ead5>. Acesso em: 28 ago. 2009. doi: 10.1016/S0044-8486(03)00069-3.

JOMORI, R.K. et al. Economic evaluation of Piaractus mesopotamicus juvenile production in different rearing systems. Aquaculture, v.243, p.175-183, 2005. Disponível em: <http:/ /www.sciencedirect.com/science/article/B6T4D-4DVBJT9-1/2/ b06c78926849fb939c3b9d3be6cd4511>. Acesso em: 28 ago. 2009. doi: 10.1016/j.aquaculture.2004.09.0340.

LUCHINI, L.M. Producción de "bagre negro" o catfish sudamericano. Revista Argentina de Producción Animal, v.8, n.5, p.433-439, 1988. Disponível em: <http:// www.produccionbovina.com.ar/produccion_peces/piscicultura/ 80-bagre.pdf>. Acesso em: 29 abr. 2005.

MAI, M.G.; ZANIBONI-FILHO, E. Efeito da idade de estocagem em tanques externos no desempenho da larvicultura do dourado Salminus brasiliensis (Osteichthyes, Characidae). Acta Scientiarum: Animal Sciences, v.27, n.2, p.287-296, 2005. Disponível em: <http://periodicos.uem.br/ojs/index.php/ ActaSciAnimSci/article/view/1252/668>. Acesso em: 28 ago. 2009. doi: $10.4025 /$ actascianimsci.v27i2.1252. 
PERSON-LE RUYET, J. et al. Marine fish larvae feeding: formulated diets or live prey? Journal World Aquaculture Society, v.24, 211-224, 1993. Disponível em: <http:// dx.doi.org/10.1111/j.1749-7345.1993.tb00010.x>. Acesso em: 22 fev. 2008. doi: 10.1111/j.1749-7345.1993.tb00010.x.

PIAIA, R.; RADÜNZ NETO, J. Efeito de níveis crescentes de levedura de álcool em rações contendo fígado bovino sobre a performance de larvas de jundiá (Rhamdia quelen). Ciência Rural, v.27, n.2, p.313-317, 1997. Disponível em: <http:// www.scielo.br/pdf/cr/v27n2/a24v27n2.pdf>. Acesso em: 15 mar. 2008. doi: 10.1590/S0103-84781997000200024.

SCHMitToU, H.R. High density fish culture in low volume cages. Singapore: American Soybean Association, 1993. 78p.
SWANN, L.D. et al. Cage culture of fish in the north central region. United States: Department of Agriculture, 1994. 14p. (Technical Bulletin Series \#110). Disponível em: $<$ http://aquanic.org/publicat/usda_rac/tr/ncrac/tb110.pdf $>$. Acesso em: 18 abr. 2007.

ULIANA, O. et al. Substituição parcial ou total de óleo de canola por lecitina de soja em rações para larvas de jundiá (Rhamdia quelen), pisces, Pimelodidae. Ciência Rural, v.31, n.4, p.677-681, 2001. Disponível em: <http://www.scielo.br/ s c i e lo.php? s c ript $=$ sci _art text \& pid $=$ S 0103 $84782001000400019 \& \operatorname{lng}=\mathrm{pt} \& \mathrm{nrm}=\mathrm{iso}>$. Acesso em: 22 abr. 2005. doi: 10.1590/S0103-84782001000400019. 